\title{
Energy efficiency of pulsed actuations on linear resonators
}

\author{
M. Domínguez \\ MNT Micro and Nano Technologies group. UPC-Campus Nord Ed. C4 \\ Jordi Girona, 1-3, 08034 Barcelona, Spain \\ e-mail: mpumar@eel.upc.edu
}

\begin{abstract}
The objective of this work is to show that, under some circumstances, the Sign of a Sampled Sinusoid sequences, briefly $S^{3}$, are optimal to provide maximum energy transfer to linear resonators in the context of discrete-time pulsed actuation at periodic times with bounded sequences. It will be proved that there is an optimal $S^{3}$ sequence which maximizes the resonator amplitude at any given finite time, and that under some conditions there is a sufficiently high time above which any $S^{3}$ sequence at the resonant frequency of the resonator also provides a locally unique maximum, in the case of a lossless or leaky resonator. The tool used to prove this last result is a theorem of quadratic programming. Since Pulsed Digital Oscillators, PDOs, under certain conditions produce $S^{3}$ sequences, a variation of the standard PDO topology that simplifies these conditions is also proposed. It is proved that except for a set of initial conditions of the resonator of zero Lebesgue measure, the bitstream at the output of this topology produces a locally unique maximum in the total energy transferred to the resonator.
\end{abstract}

Index Terms - Energy efficiency, resonators, oscillators, sigmadelta modulation.

\section{List of symbols}

$\begin{array}{cl}\langle a\rangle & a \text { Modulus } 1, a \in \mathbb{R} \\ \lfloor a\rfloor & \text { Largest integer less or equal to } a, a \in \mathbb{R} \\ z^{*} & \text { Complex conjugate of } z \in \mathbb{C} \\ R_{S}^{n \times n} & \text { Space of symmetric real matrices } \\ & \text { of dimension } n \times n \\ v^{T} & \text { Transpose of } v \in \mathbb{R}^{n} \\ \operatorname{sgn}(a) & \text { Sign function, } a \in \mathbb{R} \\ \operatorname{Re}(z) & \text { Real part of } z \in \mathbb{C} \\ \operatorname{Im}(z) & \text { Imaginary part of } z \in \mathbb{C} \\ \text { g.c.d. }(M, N) & \text { Greater common divider }, M, N \in \mathbb{N}\end{array}$

\section{INTRODUCTION}

The actuation of Microelectromechanical Systems (MEMS), such as resonators, with short pulses of force is common, [1]-[2]. Among other advantages it allows to overcome some of the nonlinearities usually present in the actuation of MEMS devices. This is for example the actuation principle used in Pulsed Digital Oscillators (PDOs), [3]-[8].

This paper is concerned with finding optimal bounded sequences to actuate linear resonators periodically with deltas and obtain maximum energy transfer. Besides its obvious appeal, the idea of maximizing the energy transfer to any given device, in particular resonators, is important for a large number of applications. For example, in [9] the idea of applying a succession of pulses to high-Q resonators is proposed to improve the wake-up time of oscillators for RF applications. The use of an optimal excitation sequence may reduce this time.

Without loss of generality we may assume that the resonator is mechanical. The equation governing the resonator and the applied excitation is of the form:

$$
x^{\prime \prime}(t)+2 \rho \omega_{0} x^{\prime}(t)+\omega_{0}^{2} x(t)=\frac{1}{m} \sum_{i=0}^{\infty} b_{i} \delta\left(t-i T_{S}\right)
$$

where $m$ is the mass of the resonator, $\omega_{0}=2 \pi f_{0}$, being $f_{0}$ the natural frequency of the resonator, $0 \leq \rho \leq 1$ is the damping factor, $T_{S}$ is the actuation period and $b_{i}$ is the amplitude of $i$-th delta applied to the resonator. The natural frequency is related to the physical parameters of the spring-mass system through $\left(2 \pi f_{0}\right)^{2}=\omega_{0}^{2}=\kappa / m$, where $\kappa$ is the spring constant.

The question this paper addresses is: what are the bounded sequences, $\left(b_{i}\right)$ with $\left|b_{i}\right| \leq 1$, that maximize the energy transfer to the resonator? It will be shown that, under some conditions, sequences of the form:

$$
b_{n}=\operatorname{sgn}(\sin (2 \pi n f+\theta)), f \in \mathbb{R}, \theta \in[0,2 \pi), n \geq 0
$$

where $f$ is the resonant frequency of the resonator, may be optimal for maximizing the energy transfer to linear resonators. This class of sequences is called the Sign of a Sampled Sinusoid, briefly $S^{3}$ sequences.

Section II states the problem in precise terms. It is shown in Section III that there is always an $S^{3}$ sequence that is the optimal actuation of a finite dimensional linear system for any given finite time. However, it is very easy to present numerical evidences showing that there is no general $S^{3}$ sequence that is a solution for all sampling times or up from any given time. Therefore, the objective of Section IV is to prove that, under some conditions, for any $S^{3}$ sequence at the resonant frequency of the resonator, there exists a sufficiently high time above which the sequence provides a locally unique maximum of the oscillation amplitude. The tool that will be used is a second order necessary and sufficient condition of quadratic programming. The results of nonlinear convex programming cannot be used in this case because the function to minimize is concave. Some examples are analyzed in 


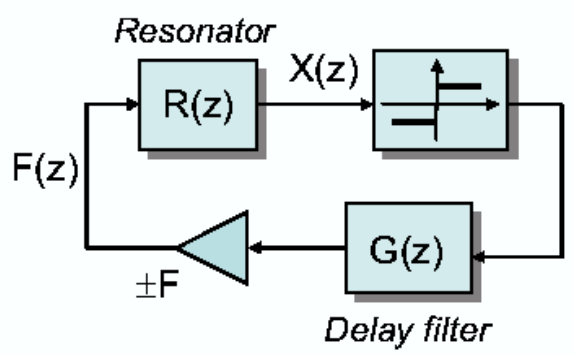

Fig. 1. General circuit topology of PDOs.

\section{Section V.}

The scheme of actuation in equation (1) is used in Pulsed Digital Oscillators. PDOs are simple circuits that allow linear resonators to maintain self-sustained oscillations. PDOs have been the object of previous analyses, [3]-[5] and experimental work, [6]-[8]. These circuits allow to monitor changes in the resonant frequency of the resonator simply by processing the binary sequence generated at their output, [6]. The circuit topology consists of a resonator, a 1-bit quantizer (sign function), and a simple digital feedback filter, $G(z)$, see Figure 1. The actuation force fed into the resonator follows equation (1) and for feedback filters of the form $G(z)=z^{-L}, L \geq 0$, the actuation is simply $b_{i}=\operatorname{sgn}\left(x\left((i-L) T_{S}\right)\right)$. The bitstream, $\left(b_{i}\right)$, at the output of the sign function is the digital output of these oscillators, and the oscillation frequency can be easily extracted from it, [4].

Under certain conditions, PDOs produce $S^{3}$ sequences with the same frequency as the resonator. For lossless resonators with rational resonant frequency, it is necessary that the oscillator is 'tuned' to this specific frequency, [4]. The 'tuning' requirement is a condition based on the rational frequency and the feedback filter of the oscillator. This is due to the fact that the resonator variable sensed in standard PDOs is the position, not the actuation variable, which is the resonator velocity. In order to overcome this requirement, and produce PDOs tuned to all rational frequencies, a variation of the standard topology of PDOs, is proposed in Section VI, namely the Direct PDOs or DPDOs. In this version, the variable of the resonator that is being sensed is the same as that on which the actuation is applied (e.g., in a mechanical resonator, the sign of the velocity). Two theorems are presented that show that under milder conditions DPDOs produce $S^{3}$ sequences at the resonant frequency of the resonator. Finally, it is also proved that DPDOs, except for a set of initial conditions of the resonator of zero Lebesgue measure, always provide a local maximum in the total energy transferred to the resonator.

\section{STATEMENT OF THE PROBlem}

The objective of this section is to define different criteria that will allow us to say that an excitation sequence is optimal. At any given time, $t$, the energy stored in the resonator is:

$$
E(t)=\frac{1}{2} \kappa x(t)^{2}+\frac{1}{2} m x^{\prime}(t)^{2}
$$

where $\kappa$ is the spring constant, $m$ is the mass of the resonator and $x(t)$ and $x^{\prime}(t)$ are the instantaneous position and velocity of the resonator respectively. On the other hand, each time a delta of force of amplitude $P$ is applied at time $t=\tau$, the increase of energy is given by, [3]:

$$
\Delta E(\tau)=\frac{P^{2}}{2 m}+P x^{\prime}(\tau)
$$

This means that each time we kick a resonator, depending on the sign and magnitude of both the excitation and the instantaneous velocity of the resonator, we may either increase or decrease its stored energy. This allows us to define different functions:

Definition 1: For any given resonator, the set of functions $E_{n}: \mathbb{R}^{2} \times \mathbb{R}^{n} \rightarrow \mathbb{R}, n \in \mathbb{N}$, is defined as:

$$
\begin{gathered}
E_{n}\left(x_{0}, v_{0}, b_{0}, \cdots, b_{n-1}\right)= \\
=E(n T s)=\frac{1}{2} \kappa x\left(n T_{S}\right)^{2}+\frac{1}{2} m x^{\prime}\left(n T_{S}\right)^{2}
\end{gathered}
$$

where $x\left(n T_{S}\right)$ and $x^{\prime}\left(n T_{S}\right)$ are the position and velocity respectively of the resonator governed by equation (1), with initial condition $\left(x_{0}, v_{0}\right)$, after $n$ deltas with amplitudes $b_{i}, i=0, \cdots, n-1$, have been applied to it.

From Definition 1 it is evident that $E_{n}$ is the final stored energy in a resonator that, from a given initial condition, is excited with $n$ deltas of force with amplitudes $b_{i}$.

Definition 2: For any given resonator, the set of functions $\Delta E_{n}: \mathbb{R}^{2} \times \mathbb{R}^{n} \rightarrow \mathbb{R}, n \in \mathbb{N}$, is defined as:

$$
\Delta E_{n}\left(x_{0}, v_{0}, b_{0}, \cdots, b_{n-1}\right)=\frac{n}{2 m}+\sum_{i=0}^{n-1} b_{i} x^{\prime}\left(i T_{S}\right)
$$

where $x\left(i T_{S}\right)$ and $x^{\prime}\left(i T_{S}\right)$ are respectively the position and velocity of the resonator governed by equation (1), with initial condition $\left(x_{0}, v_{0}\right)$, after $n$ deltas with amplitudes $b_{i}, i=0, \cdots, n-1$, have been applied to it.

The functions $\Delta E_{n}$ represent the energy that has been introduced into a resonator that, from a given initial condition, is excited with $n$ deltas of force of amplitudes $b_{i}$.

Now, we may define two different optimization problems:

(P1) Given a resonator and its initial condition, $\left(x_{0}, v_{0}\right)$, we say that a bounded sequence $\left(b_{i}\right),\left|b_{i}\right| \leq 1$, provides a global (or local) maximum of energy at time $n$ from $\left(x_{0}, v_{0}\right)$, if it is a global (or local) maximum of $E_{n}$.

(P2) Given a resonator and its initial condition, $\left(x_{0}, v_{0}\right)$, we say that a bounded sequence $\left(b_{i}\right),\left|b_{i}\right| \leq 1$, provides a global (or local) maximum of energy transfer up to time $n$ from $\left(x_{0}, v_{0}\right)$, if it is a global (or local) maximum of $\Delta E_{n}$.

In both cases, the maxima must be understood as maxima of $E_{n}$ or $\Delta E_{n}$ seen as functions of the amplitudes of the 
deltas, $\left(b_{i}\right)$, for an initial condition of the resonator $\left(x_{0}, v_{0}\right)$. In the case of a lossless resonator, $\rho=0$, it is evident that both problems are equivalent, since:

$$
\begin{gathered}
E_{n}\left(x_{0}, v_{0}, b_{0}, \cdots, b_{n-1}\right)= \\
=\frac{1}{2} \kappa x_{0}^{2}+\frac{1}{2} m v_{0}^{2}+\Delta E_{n}\left(x_{0}, v_{0}, b_{0}, \cdots, b_{n-1}\right)
\end{gathered}
$$

because between the application of any two consecutive deltas the energy stored in the resonator is conserved.

The time evolution of a resonator obeying equation (1), with an adequate change of variables:

$$
\begin{aligned}
& x_{n}=x\left(n T_{S}\right) \\
& v_{n}=\frac{1}{\sqrt{1-\rho^{2}}}\left(\rho x\left(n T_{S}\right)+\frac{x^{\prime}\left(n T_{S}\right)}{\omega_{0}}\right)
\end{aligned}
$$

is simply described by:

$$
\left(\begin{array}{c}
x_{i+1} \\
v_{i+1}
\end{array}\right)=\alpha A\left(\begin{array}{c}
x_{i} \\
v_{i}
\end{array}\right)+F\left(\begin{array}{c}
0 \\
b_{i}
\end{array}\right)
$$

with:

$$
\begin{aligned}
A & =\left(\begin{array}{cc}
\cos 2 \pi f & \sin 2 \pi f \\
-\sin 2 \pi f & \cos 2 \pi f
\end{array}\right) \\
\alpha & =e^{-2 \pi \frac{\rho}{\sqrt{1-\rho^{2}}} f} \\
F & =\frac{1}{m \omega_{0} \sqrt{1-\rho^{2}}} \\
f & =\frac{f_{0}}{f_{S}} \sqrt{1-\rho^{2}}
\end{aligned}
$$

This means that:

$$
\begin{gathered}
\left(\begin{array}{c}
x_{n} \\
v_{n}
\end{array}\right)-\alpha^{n} A^{n}\left(\begin{array}{c}
x_{0} \\
v_{0}
\end{array}\right)= \\
=F(\alpha A)^{n-1} \sum_{i=0}^{n-1}(\alpha A)^{-i}\left(\begin{array}{c}
0 \\
b_{i}
\end{array}\right)=\sum_{i=0}^{n-1} b_{i} \nu_{i}
\end{gathered}
$$

where the vectors $\nu_{i}$ are defined as $\nu_{i}=$ $F(\alpha A)^{n-1-i}\left(\begin{array}{c}0 \\ 1\end{array}\right), i=0, \ldots, n-1$.

This allows us to state another optimization problem:

(P3) Given a resonator and its initial condition, $\left(x_{0}, v_{0}\right)$, we say that a bounded sequence $\left(b_{i}\right),\left|b_{i}\right| \leq 1$, provides a global (or local) maximum of amplitude at time $n$ from $\left(x_{0}, v_{0}\right)$, if it is a global (or local) maximum of $\left\|\left(x_{n}, v_{n}\right)\right\|$.

Note that $\|\cdot\|$ is the Euclidean norm associated with the standard inner product $\langle\cdot, \cdot\rangle$.

It is easy to see that problems P1-P3 are equivalent for any lossless resonator. On the other hand, they depend on the initial condition of the resonator and are specified for a given time, $n$. What is really interesting is to analyze whether a given sequence is a global (or local) solution of any of those problems asymptotically (up from a sufficiently high time), and regardless of the initial condition of the resonator.
We may now define:

Definition 3: Given a resonator and its initial condition, $\left(x_{0}, v_{0}\right)$, we say that a bounded sequence is asymptotically a global (or local) solution of problems P1, P2 or P3 if there is a $k>0$ such that for $n>k$ the sequence is a solution of problems $\mathrm{P} 1, \mathrm{P} 2$ or $\mathrm{P} 3$.

In order to exclude the influence of the initial condition of the resonator, and also to simplify the problem at some points, we will use the following functions $H_{n}: \mathbb{R}^{n} \rightarrow \mathbb{R}$ :

$$
H_{n}\left(b_{0}, \ldots, b_{n-1}\right)=\left\|\sum_{i=0}^{n-1} b_{i} \nu_{i}\right\|^{2}
$$

For a lossless resonator, it is $\frac{\kappa}{2} H_{n}\left(b_{0}, \ldots, b_{n-1}\right)=$ $E_{n}\left(0,0, b_{0}, \ldots, b_{n-1}\right)=\Delta E_{n}\left(0,0, b_{0}, \ldots, b_{n-1}\right)$, where $\kappa$ is the spring constant of the resonator.

The optimization of the actuation control of linear and nonlinear systems is a wide field, [10]-[11]. We will first analyze the following problem:

$$
\begin{gathered}
\text { Minimize }-H_{n}\left(b_{0}, \ldots, b_{n-1}\right) \\
\text { subject to }\left|b_{0}\right| \leq 1, \ldots,\left|b_{n-1}\right| \leq 1
\end{gathered}
$$

The goal is:

a) to find the bounded sequence $\left(b_{n}\right),\left|b_{n}\right| \leq 1$, maximizing $H_{n}$,

b) to determine if this result can be extended for $n \rightarrow \infty$,

c) and to analyze when b) implies that the sequence solves asymptotically problems P1, P2 or P3 for any initial condition of the resonator.

\section{III. $S^{3}$ SEQUENCES AND DISCRETE-TIME FINITE} DIMENSIONAL LINEAR SYSTEMS: GLOBAL MAXIMUM OF $H_{n}$ FOR FINITE TIME

Given a finite dimensional linear function, $h: \mathbb{R}^{n} \rightarrow \mathbb{R}^{d}$ :

$$
h_{n}\left(b_{0}, \ldots, b_{n-1}\right)=\sum_{i=0}^{n-1} \nu_{i} b_{i}
$$

with $\nu_{0}, \ldots, \nu_{n-1} \in \mathbb{R}^{d}$ what are the global optimum bounded values of $b_{1}, \ldots, b_{n}$ that maximize $\left\|h_{n}\right\|$ ? Note that $\|u\|=\max _{\|z\|=1}\langle u, z\rangle$. Here $\max _{\|z\|=1}\langle u, z\rangle$ stands for the maximum of $\langle u, z\rangle$, as a function of $z$, subjected to $\|z\|=1$. For $d=1$ (scalar case), clearly the solution is given by $b_{i}=\operatorname{sgn}\left(\nu_{i}\right)$ along with the "mirror" solution $b_{i}=-\operatorname{sgn}\left(\nu_{i}\right)$. For a general dimension $d$, we have: 


$$
\begin{aligned}
\max _{\left|b_{i}\right| \leq 1}\left\|\sum_{i=1}^{n} b_{i} \nu_{i}\right\| & =\max _{\left|b_{i}\right| \leq 1} \max _{\|z\|=1}\left\langle\sum_{i=1}^{n} b_{i} \nu_{i}, z\right\rangle \\
& =\max _{\|z\|=1} \max _{\left|b_{i}\right| \leq 1} \sum_{i=1}^{n} b_{i}\left\langle\nu_{i}, z\right\rangle \\
& =\max _{\|z\|=1} \sum_{i=1}^{n}\left|\left\langle\nu_{i}, z\right\rangle\right|
\end{aligned}
$$

where in the last step we have used the solution for $d=1$ so that $b_{i}=\operatorname{sgn}\left(\left\langle\nu_{i}, z\right\rangle\right)$. The $z$ that maximizes this final expression will determine the sequence of $b_{i}$ that maximizes the first expression.

Now it must be noted that:

$$
\begin{gathered}
\operatorname{sgn}\left(\left\langle\nu_{i}, z\right\rangle\right)=\operatorname{sgn}\left(\left\langle A^{n-1-i}\left(\begin{array}{l}
0 \\
1
\end{array}\right), z\right\rangle\right)= \\
=\operatorname{sgn}(\cos (2 \pi f i+\theta))
\end{gathered}
$$

for a certain angle $\theta$. This means, that for any problem of the form (13), and any given time $n$, there is an $S^{3}$ sequence, with the same frequency as the resonator, that is the solution of the optimization problem. It is very easy, though, to find numerical evidences showing that in general this solution is not valid for another time $n^{\prime} \neq n$. However, in the following section we will prove that, under some conditions, any $S^{3}$ sequence with the same frequency as the resonator is a locally unique maximum of problem (13).

It is important to note that, if we apply this result to linear resonators, the conclusion is valid for any frequency (rational or irrational) and any damping losses.

\section{UNIQUE LOCAL SOLUTIONS APPLYING QUADRATIC PROGRAMMING RESULTS}

\section{A. Theorem by Mangarasian and Contesse}

In order to prove that $S^{3}$ sequences may be local solutions of problem (13) we will use a result by Mangarasian and Contesse, whose statement and proof can be found in [12], section 3.2, or in [13]. The problem to solve is the following:

\section{Problem}

Let $D \in R_{S}^{n \times n}, B \in R^{m \times n}, C \in R^{s \times n}, c \in R^{n}, a \in R^{m}$ and $d \in R^{s}$. The problem is:

$$
\min _{x \in R^{n}}\left\{\frac{1}{2} x^{T} D x+c^{T} x: x \in R^{n}, B x \geq a, C x=d\right\}
$$

The statement of the theorem by Mangarasian and Contesse is the following:

Theorem (Mangarasian and Contesse (1980)): The necessary and sufficient condition for a point $\bar{x} \in R^{n}$ to be a locally unique solution of problem (17) is that there exists a pair of vectors

$$
(\lambda, \mu)=\left(\lambda_{1}, \ldots, \lambda_{m}, \mu_{1}, \ldots, \mu_{S}\right) \in R^{m} \times R^{S}
$$

such that

i) the system

$$
\left\{\begin{array}{l}
D \bar{x}-B^{T} \lambda-C^{T} \mu+c=0, \lambda \geq 0 \\
B \bar{x}-a \geq 0, C \bar{x}=d \\
\lambda^{T}(B \bar{x}-a)=0
\end{array}\right.
$$

is satisfied, and

ii) if $\zeta \in R^{n} \backslash\{0\}$ is such that $B_{I_{1}} \zeta=0, B_{I_{2}} \zeta \geq 0, C \zeta=0$, where

$$
\begin{aligned}
& I_{1}=\left\{i: B_{i} \bar{x}=a_{i}, \lambda_{i}>0\right\}, I_{2}=\left\{i: B_{i} \bar{x}=a_{i}, \lambda_{i}=0\right\} \\
& \text { then } \zeta^{T} D \zeta>0 .
\end{aligned}
$$

The terms $B_{I_{1}}$ or $B_{I_{2}}$, indicate the matrices formed by row vectors of $B$ with indices in the sets $I_{1}$ or $I_{2}$, respectively.

The first condition, i), ensures that the candidate solution, $\bar{x}$, is a Karush-Kuhn-Tucker point, which is defined in the optimization theorem of the same name. The second condition, ii), is a second order condition that ensures locally unique optimality in the case of quadratic programming. If, in condition ii), the term $\zeta^{T} D \zeta>0$ is changed to $\zeta^{T} D \zeta \geq 0$ then the uniqueness of the local solution is no longer guaranteed, [12].

In the case of problem (13), the matrix $D$ is formed by $D_{i j}=-2\left\langle\nu_{i}, \nu_{j}\right\rangle$, which is symmetric. It must be noted, that this matrix is negative definite, and therefore all the solutions will lie on the boundary.

The constraint matrix of the theorem, $B \in \mathbb{R}^{2 n \times n}$, may be constructed as: $B_{i, i}=-1, i=0, \ldots, n-1$, and $B_{i, i-n}=1, i=n, \ldots, 2 n-1$, with all other terms zero. With regard to vector $a \in \mathbb{R}^{2 n}$, it is $a=(-1, \ldots,-1)^{T}$, and vectors $C, c$ and $d$ are zero in our case.

\section{B. Application of the theorem}

Now, conditions (19) translated to our problem imply to find vectors $(\mathbf{b}, \lambda)=\left(b_{0}, \ldots, b_{n-1}, \lambda_{0}, \ldots, \lambda_{n-1}, \lambda_{0}^{\prime}, \ldots, \lambda_{n-1}^{\prime}\right)$ such that for $k=0, \ldots, n-1$ :

$$
\begin{aligned}
-2 \sum_{i=0}^{n-1}\left\langle\nu_{k}, \nu_{i}\right\rangle b_{i}+\lambda_{k}-\lambda_{k}^{\prime} & =0 \\
\left|b_{k}\right| & \leq 1 \\
\lambda_{k}\left(-b_{k}+1\right) & =0 \\
\lambda_{k}^{\prime}\left(b_{k}+1\right) & =0
\end{aligned}
$$


with $\lambda_{0}, \ldots, \lambda_{n-1}, \lambda_{0}^{\prime}, \ldots, \lambda_{n-1}^{\prime} \geq 0$. It must be noted that we have $2 n$ constraints.

Let us assume for a moment that a binary sequence, $\left(b_{n}\right)$, with $\left|b_{n}\right|=1, n \geq 0$, is a solution of the above conditions (21). Then, if $\sum_{i=0}^{n-1}\left\langle\nu_{k}, \nu_{i}\right\rangle b_{i} \neq 0$, condition ii) of the theorem is satisfied. This is because if this condition is met, the set $\left\{i \bmod n: i \in I_{1}\right\}$ is $\{0, \ldots, n-1\}$, or what is the same, for each $k=0, \ldots, n-1$ it is either $\lambda_{k}>0$ or $\lambda_{k}^{\prime}>0$. Therefore, the only $\zeta \in R^{n}$ such that $B_{I_{1}} \zeta=0$ is $\zeta=0$, i.e., the solution lies entirely on the set of vertices of the (n-dimensional) constraining cube.

Furthermore, due to the fact that in this problem the matrix $D$ is negative definite it is not possible to find any vector $\zeta$, other than 0 , satisfying the condition $\zeta^{T} D \zeta \geq 0$. This, together with the fact that the theorem provides sufficient and necessary conditions for optimality, ensures that any solution of the problem must necessarily be binary, and $\sum_{i=0}^{n-1}\left\langle\nu_{k}, \nu_{i}\right\rangle b_{i} \neq 0$ (if not the index set of the theorem, $I_{2}$, may not be empty and in any case $\left.\left\{i \bmod n: i \in I_{1}\right\} \neq\{0, \ldots, n-1\}\right)$. This allows us to state the following result.

Lemma 1: The necessary and sufficient condition for a given bounded sequence, $\left(b_{0}, \ldots, b_{n-1}\right),\left|b_{i}\right| \leq 1,0 \leq i<n$, to be a local solution of problem (13), is that:

$$
\operatorname{sgn}\left(\sum_{i=0}^{n-1}\left\langle\nu_{k}, \nu_{i}\right\rangle b_{i}\right)=b_{k} \neq 0
$$

for $k=0, \ldots, n-1$.

Note that in the above statement it is assumed that $\operatorname{sgn}(0)=0$.

Obviously any local solution of the problem is locally unique, and:

Corollary 1: Any local solution of problem (13) is an $S^{3}$ sequence at the resonant frequency of the resonator.

Proof. Any binary sequence satisfying the conditions of Lemma 1 for a certain time $n$, will be of the form:

$$
b_{i}=\operatorname{sgn}\left(\left\langle\nu_{i}, z\right\rangle\right), 0 \leq i<n
$$

where $z=\sum_{k=0}^{n-1} \nu_{k} b_{k}$. Therefore, as indicated by equation (16), this is an $S^{3}$ sequence at the resonant frequency of the resonator.

\section{Complex notation}

In order to see that $S^{3}$ sequences at the resonant frequency of the resonator are a solution of the problem we will generate an auxiliary complex sequence $u_{n}=x_{n}+j v_{n}$, where $x_{n}$ and $v_{n}$ are defined as in (8). This will allow us to use the calculation of the Fourier Transform of $S^{3}$ sequences, which can be found in [4], in order to see if they are a solution to problem (13).

If we define $p \in \mathbb{C}$ as $p=e^{-j 2 \pi f}$, with this notation, equation (10) becomes:

$$
u_{n+1}=\alpha p u_{n}+j F b_{n}, \quad n \geq 0
$$

and we may also define an auxiliary sequence, $h_{n}\left(b_{0}, \ldots, b_{n-1}\right)$ as:

$$
\begin{gathered}
h_{n}\left(b_{0}, \ldots, b_{n-1}\right)=u_{n}-\alpha^{n} p^{n} u_{0}= \\
=j F(\alpha p)^{n-1} \sum_{i=0}^{n-1} \alpha^{-i} p^{-i} b_{i}
\end{gathered}
$$

It must be noted that $H_{n}\left(b_{0}, \ldots, b_{n-1}\right)$, as defined in (12), is simply $\left|h_{n}\left(b_{0}, \ldots, b_{n-1}\right)\right|^{2}$. Finally, if we further define:

$$
G_{n}\left(b_{0}, \ldots, b_{n-1}\right)=\sum_{i=0}^{n-1} \alpha^{-i} p^{-i} b_{i}
$$

then, the problem of maximizing $H_{n}\left(b_{0}, \ldots, b_{n-1}\right)$, is equivalent to the maximization of $\left|G_{n}\left(b_{0}, \ldots, b_{n-1}\right)\right|^{2}$.

Finally, any $S^{3}$ sequence at the resonant frequency of the resonator may be expressed as $b_{k}=\operatorname{sgn}\left(\operatorname{Re}\left(p^{k} z_{0}\right)\right)=$ $\operatorname{sgn}\left(\operatorname{Re}\left(p^{k} z_{0}\right)\right), k \geq 0$ for a certain $z_{0} \in \mathbb{C}$, and $\left\langle\nu_{k}, \nu_{m}\right\rangle=$ $\operatorname{Re}\left(F^{2} \alpha^{(2 n-2-k-m)} p^{k-m}\right)$. Now, condition (22) of Lemma 1 may be stated for our problem as:

$$
\operatorname{sgn}\left(\operatorname{Re}\left(p^{k} G_{n}(\mathbf{b})\right)\right)=b_{k} \neq 0
$$

for $k=0, \ldots, n-1$, with $\mathbf{b}=\left(b_{0}, \ldots, b_{n-1}\right)$.

NOTATION: To simplify notation, from now onwards, we will refer to $G_{n}(\mathbf{b})$ as $G_{n}$, being b an $S^{3}$ sequence.

D. Case $\alpha=1, p=e^{-j 2 \pi f}, f=\frac{M}{N} \in \mathbb{Q}$, g.c.d. $(M, N)=1$

Now, we will see if any binary $S^{3}$ sequence at the resonant frequency of the resonator, $b_{n}=\operatorname{sgn}\left(\operatorname{Re}\left(p^{n} z_{0}\right)\right), z_{0} \in \mathbb{C}$, is a locally unique solution of problem (13). In order to see if the term $G_{n}$ generated by the above sequence fulfills the conditions of Lemma 1, we calculate first $G_{N}$. Now, see the Appendix, for $N$ even it is:

$$
G_{N}=j 2 e^{-j \frac{\pi}{N}} \frac{e^{-j 2 \pi \frac{\lfloor\beta N\rfloor}{N}}}{\sin \left(\frac{\pi}{N}\right)},
$$

and for $N$ odd:

$$
G_{N}=j e^{-j \frac{\pi}{2 N}} \frac{e^{-j 2 \pi \frac{\lfloor 2 \beta N\rfloor}{2 N}}}{\sin \left(\frac{\pi}{2 N}\right)}
$$

where $\beta=1 / 4-\arg \left(z_{0}\right) / 2 \pi$. In order to satisfy requirement (27) for $n=N$, it is necessary and sufficient that:

$$
\operatorname{sgn}\left(\operatorname{Re}\left(p^{n} G_{N}\right)\right)=b_{n}=\operatorname{sgn}\left(\operatorname{Re}\left(p^{n} z_{0}\right)\right) \neq 0
$$


for $n=0, \cdots, N-1$. It can be easily checked that for $N$ even this condition is equivalent to:

$$
\left\langle\frac{\left\lfloor\left(\frac{1}{4}-\frac{\arg \left(G_{N}\right)}{2 \pi}\right) N\right\rfloor}{N}\right\rangle=\left\langle\frac{\lfloor\beta N\rfloor}{N}\right\rangle
$$

and that expression (28) satisfies it. On the other hand, for $N$ odd, condition (30) is equivalent to:

$$
\left\langle\frac{\left\lfloor\left(\frac{1}{4}-\frac{\arg \left(G_{N}\right)}{2 \pi}\right) 2 N\right\rfloor}{2 N}\right\rangle=\left\langle\frac{\lfloor 2 \beta N\rfloor}{2 N}\right\rangle
$$

and this condition is also satisfied by expression (29). Both these conditions are equivalent to the 'tuning' condition as defined in [4].

On the other hand, due to the fact that $p^{N}=1$ and $\alpha=1$ :

$$
G_{n}=G_{n \bmod N}+\left\lfloor\frac{n}{N}\right\rfloor G_{N}, \quad n \geq 0
$$

and therefore:

$$
\lim _{n \rightarrow \infty} \frac{N}{n} G_{n}=G_{N}
$$

Finally, due to the fact that for $N$ even:

$$
\frac{\left\langle\left(\frac{1}{4}-\frac{\arg \left(G_{N}\right)}{2 \pi}\right) N\right\rangle}{N}=\frac{1}{2 N},
$$

and for $N$ odd:

$$
\frac{\left\langle\left(\frac{1}{4}-\frac{\arg \left(G_{N}\right)}{2 \pi}\right) 2 N\right\rangle}{2 N}=\frac{1}{4 N},
$$

then there is a sufficiently large number $k_{0}(f)$ such that for any $n>k_{0}(f)$ we have that

$$
\operatorname{sgn}\left(\operatorname{Re}\left(p^{k} z_{0}\right)\right)=\operatorname{sgn}\left(\operatorname{Re}\left(p^{k} G_{n}\right)\right), k \geq 0 .
$$

This allows us to state the following results:

Theorem 1: For any lossless resonator with rational frequency, there is $k_{0}(f)>0$ such that any $S^{3}$ sequence at the resonant frequency of the resonator is a locally unique solution of (13), for $n>k_{0}(f)$.

Corollary 2: For any lossless resonator with rational frequency, regardless of its initial condition, any $S^{3}$ sequence at the resonant frequency of the resonator is asymptotically a locally unique solution of problems $\mathrm{P} 1, \mathrm{P} 2$ and $\mathrm{P} 3$.

Proof. From equation (34) it is evident that if we add the initial condition of the resonator to $G_{n}$, the conditions to apply Lemma 1 still hold. Therefore, asymptotically the sequence is a unique solution of problem P3, and because it is a lossless resonator, also of problems $\mathrm{P} 1$ and $\mathrm{P} 2$.

\section{E. Case $\alpha=1, p=e^{-j 2 \pi f}, f \in \mathbb{R} \backslash \mathbb{Q}$}

In this case it is not easy to apply Lemma 1 in the same way. On the other hand, for all bounded complex sequences, $\left(a_{i}\right)$, the Lebesgue measure in $[0,1]$ of all frequencies such that:

$$
\lim _{n \rightarrow \infty} \frac{1}{n}\left|\sum_{i=0}^{n-1} a_{i} e^{-j 2 \pi f i}\right|>0
$$

is zero. This can be easily checked because, following a similar procedure as in Lemma 4.1 of [14], if $C_{1}=\{z \in \mathbb{C}$ : $|z|=1\},\left(a_{i}\right)$ is any bounded complex sequence, $d \nu$ is the Lebesgue measure on $C_{1}$, and $d z$ being the standard complex integration:

$$
\begin{gathered}
\int_{C_{1}}\left|\sum_{i=0}^{n-1} a_{i} \nu^{i}\right|^{2} d \nu=\sum_{l=0}^{n-1} \sum_{i=0}^{n-1} a_{i} a_{l}^{*} \int_{C_{1}} z^{i} z^{-l}\left(-j z^{-1}\right) d z= \\
\sum_{i=0}^{n-1}\left|a_{i}\right|^{2} \int_{C_{1}}\left(-j z^{-1}\right) d z=2 \pi \sum_{i=0}^{n-1}\left|a_{i}\right|^{2}=O(n)
\end{gathered}
$$

and therefore for almost all $z \in C_{1}$ it is $\left|\sum_{i=0}^{n-1} a_{i} z^{i}\right|^{2}=$ $O(n)$, with respect to the Lebesgue measure on $C_{1}$, and therefore:

$$
\lim _{n \rightarrow \infty} \frac{1}{n}\left|\sum_{i=0}^{n-1} a_{i} e^{-j 2 \pi f i}\right|=0
$$

except for a set of frequencies of zero Lebesgue measure in $[0,1]$.

On the other hand, due to the fact that for a sequence $s=$ $\left(\operatorname{sgn}\left(\operatorname{Re}\left(p^{i} z_{0}\right)\right)\right), i \geq 0, z_{0} \in \mathbb{C}$ of irrational frequency $f$, it is $\left\{\left\langle n f+\frac{\arg \left(z_{0}\right)}{2 \pi}\right\rangle: n \geq 0\right\}$ dense in $[0,1]$ and applying the Ergodic Theorem we obtain:

$$
\begin{gathered}
\frac{1}{z_{0}^{*}} \lim _{n \rightarrow \infty} \frac{1}{n} \sum_{i=0}^{n-1} p^{-i} z_{0}^{*} \operatorname{sgn}\left(\operatorname{Re}\left(p^{i} z_{0}\right)\right)= \\
\quad=2 \int_{-\frac{1}{4}}^{\frac{1}{4}} e^{j 2 \pi f} d f=\frac{2}{\pi} e^{j \arg \left(z_{0}\right)} .
\end{gathered}
$$

This means that for almost all frequencies and all bounded complex sequences, limit (38) is 0, whereas at the same time, $S^{3}$ sequences with the same frequency as the lossless resonator-like system, rational or irrational, produce an unbounded output of order $O(n)$.

\section{F. Case $f=\frac{M}{N}, \alpha \neq 1$}

In this case, and for an $S^{3}$ sequence of the same form as in (68), due to the continuity of $G_{N}$ as a function of $\alpha$ and because for $\alpha=1$ equations (35) or (36) apply, there is a number $\epsilon_{0}(f)>0$, defined as the supremum of all values of $\epsilon$ such that for $\alpha \in[1-\epsilon, 1+\epsilon]$ expressions (31) or (32) continue to hold.

On the other hand, if $\alpha \neq 1$ : 


$$
G_{n}=a_{n} G_{N}+b_{n} G_{n \bmod N}
$$

where:

$$
\begin{aligned}
& a_{n}=\frac{1-\alpha^{-\left\lfloor\frac{n}{N}\right\rfloor N}}{1-\alpha^{-N}} \\
& b_{n}=\alpha^{-\left\lfloor\frac{n}{N}\right\rfloor N}
\end{aligned}
$$

and taking into account that:

$$
\lim _{n \rightarrow \infty, \alpha \rightarrow 1} \frac{1}{a_{n}} G_{n}=G_{N}
$$

then, there is a $0<\epsilon_{1}(f) \leq \epsilon_{0}(f)$, such that for $\alpha \in(1-$ $\left.\epsilon_{1}(f), 1+\epsilon_{1}(f)\right)$ there is a $n_{0}(f, \alpha)$ such that for $n>k_{0}(f, \alpha)$ it is, for $N$ even:

$$
\left(\frac{1}{4}-\frac{\arg \left(G_{n}\right)}{2 \pi}\right) \neq \frac{\left\lfloor\left(\frac{1}{4}-\frac{\arg \left(G_{n}\right)}{2 \pi}\right) N\right\rfloor}{N}=\frac{\lfloor\beta N\rfloor}{N}
$$

For $n>n_{0}(f, \alpha)$, and $N$ odd:

$$
\left(\frac{1}{4}-\frac{\arg \left(G_{n}\right)}{2 \pi}\right) \neq \frac{\left\lfloor\left(\frac{1}{4}-\frac{\arg \left(G_{n}\right)}{2 \pi}\right) 2 N\right\rfloor}{2 N}=\frac{\lfloor 2 \beta N\rfloor}{2 N}
$$

This means, in both cases, that for $n>n_{0}(f, \alpha)$ :

$$
\operatorname{sgn}\left(\operatorname{Re}\left(p^{k} z_{0}\right)\right)=\operatorname{sgn}\left(\operatorname{Re}\left(p^{k} G_{n}\right)\right), \quad k \geq 0
$$

This, together with Theorem 1 to include the case $\alpha=1$, allows us to state the following result:

Theorem 2: For any leaky resonator with rational frequency, $f$, there is a number, $\epsilon_{1}(f)>0$, such that for any resonator with $\alpha \in\left(1-\epsilon_{1}(f), 1+\epsilon_{1}(f)\right)$ there is a $n_{0}(f, \alpha)>0$ such that any $S^{3}$ sequence at the resonant frequency of the resonator is a locally unique solution of problem (13), for $n \geq n_{0}(f, \alpha)$.

Corollary 3: For any leaky resonator with rational frequency, $f$, there is a number, $\epsilon_{2}(f)>0$, such that for any resonator with $\alpha \in\left(1-\epsilon_{2}(f), 1\right)$, any $S^{3}$ sequence at the resonant frequency of the resonator is asymptotically a locally unique solution of problems $\mathrm{P} 2$ and $\mathrm{P} 3$.

Proof. Problem P3: for $\alpha \in\left(1-\epsilon_{1}(f), 1\right)$, retaking expression (25):

$$
\begin{gathered}
u_{n+1}=(\alpha p) u_{n}+j F b_{n}= \\
=\left((\alpha p)^{n+1} u_{0}+j \alpha^{n} F p^{n} G_{n}\right)+j F b_{n}
\end{gathered}
$$

and taking into account Theorem 2, for a sufficiently high time, $n_{0}$, sufficiently low losses and rational frequency it will be for any $S^{3}$ sequence at the rational frequency of the resonator:

$$
\operatorname{sgn}\left(\operatorname{Im}\left((\alpha p)^{n+1} u_{0}+j \alpha^{n} F p^{n} G_{n}\right)\right)=\operatorname{sgn}\left(\operatorname{Re}\left(p^{n} G_{n}\right)\right)=b_{n}
$$

for each $n>n_{0}$, and therefore the sequence is a locally unique solution of problem P3, regardless of the initial condition of the resonator. This is basically due to the fact that the term associated with the initial conditions of the resonator decays exponentially.

Problem P2: if a binary sequence, $\left(b_{i}\right),\left|b_{i}\right|=1, i \geq 0$ is such that $b_{i}=\operatorname{sgn}\left(x^{\prime}\left(i T_{S}\right)\right) \neq 0$, then it is a locally unique solution of problem P2. This in fact would reflect the situation on which each delta contributes to increase the velocity of the resonator.

Now, if we prove that for sufficiently low losses it is for $n>n_{0}$ :

$$
\begin{gathered}
\operatorname{sgn}\left(v_{n}\right)=\operatorname{sgn}\left(\rho x\left(n T_{S}\right)+x^{\prime}\left(n T_{S}\right) / \omega_{0}\right)= \\
=\operatorname{sgn}\left(x^{\prime}\left(n T_{S}\right)\right) \neq 0
\end{gathered}
$$

then the sequence will be asymptotically a solution of problem P2. Now, taking into account that the resonant frequency is rational, and expression (44), we may see that the system will have a limit cycle of $N$ points. Due to the fact that this number is finite we may find a sufficiently low damping factor $\rho$, such that expression (50) holds for $n>n_{0}$. The fact that $\operatorname{sgn}\left(x^{\prime}\left(n T_{S}\right)\right) \neq 0$ is guaranteed because of the inequalities in expressions (45) or (46). Therefore the sequence is asymptotically also a solution of problem $\mathrm{P} 2$.

\section{G. Final note}

One last important point to analyze is whether with a similar approach to that used in Section III, the results obtained in this Section might have been achieved in a more straightforward manner. Now, for any bounded sequence, $(\mathbf{b}),\left|b_{i}\right| \leq 1$, it is:

$$
\begin{gathered}
\left|G_{n}(\mathbf{b})\right|=\max _{\chi \in \mathbb{C},|\chi|=1} \operatorname{Re}\left(G_{n}(\mathbf{b}) \chi^{*}\right)= \\
=\operatorname{Re}\left(G_{n}(\mathbf{b}) z^{*}\right)=\sum_{i=0}^{n-1} b_{i} \alpha^{-i} \operatorname{Re}\left(p^{-i} z^{*}\right) \leq \\
\leq \sum_{i=0}^{n-1} \operatorname{sgn}\left(\operatorname{Re}\left(p^{i} z\right)\right) \alpha^{-i} \operatorname{Re}\left(p^{-i} z^{*}\right)=\sum_{i=0}^{n-1} \alpha^{-i}\left|\operatorname{Re}\left(p^{-i} z^{*}\right)\right|
\end{gathered}
$$

for a certain $z \in \mathbb{C},|z|=1$. This suggests a procedure to prove locally unique optimality of $S^{3}$ sequences.

If a sequence of the form $\left(\mathbf{b}_{z_{0}}\right)=\operatorname{sgn}\left(\operatorname{Re}\left(p^{i} z_{0}\right)\right), z_{0} \in \mathbb{C}$, is such that:

i) $\left|G_{n}\left(\mathbf{b}_{z_{0}}\right)\right|=\operatorname{Re}\left(G_{n}\left(\mathbf{b}_{z_{0}}\right) z_{0}^{\prime *}\right)$, for a certain $z_{0}^{\prime} \in \mathbb{C}$, $\left|z_{0}^{\prime}\right|=1$, 
ii) and $\operatorname{sgn}\left(\operatorname{Re}\left(p^{i} z_{0}\right)\right)=\operatorname{sgn}\left(\operatorname{Re}\left(p^{i} z_{0}^{\prime}\right)\right) \neq 0, i=$ $0, \ldots, n-1$,

then:

$$
\left|G_{n}(\mathbf{b})\right|=\operatorname{Re}\left(G_{n}\left(\mathbf{b}_{z_{0}}\right) z_{0}^{\prime *}\right)=\sum_{i=0}^{n-1} \alpha^{-i}\left|\operatorname{Re}\left(p^{-i} z_{0}^{\prime *}\right)\right|
$$

and therefore, there is a neighborhood of the point $\left(\operatorname{sgn}\left(\operatorname{Re}\left(z_{0}\right)\right), \ldots, \operatorname{sgn}\left(\operatorname{Re}\left(p^{n-1} z_{0}\right)\right) \in \mathbb{R}^{n}\right.$, restricted to the constraining cube, where the $S^{3}$ sequence presents a locally unique solution. The last inequality of condition ii) is important to ensure that this is an optimal local solution. If it is not satisfied then, for any neighborhood of the solution, there would be points with different admissible binary sequences. This is because their $z$ maximizing (51) would generate a different sequence of bits. Therefore the solution in that case would not be a local maximum.

This procedure is equivalent to that used in [3] to show optimality of $S^{3}$ sequences for a reduced number of frequencies, and requires exactly the same calculations as the ones used in Section III, basically due to condition ii).

Nonetheless, this procedure allows us to tackle the irrational frequency case for lossless resonators, $\alpha=1$. If we define $g_{\infty}(\mathbf{b})$ as:

$$
g_{\infty}(\mathbf{b})=\lim _{n \rightarrow \infty} \frac{1}{n}\left|G_{n}(\mathbf{b})\right|
$$

and taking into account (41), then, for any $S^{3}$ sequence of irrational frequency, $\mathbf{b}_{z_{0}}=\operatorname{sgn}\left(\operatorname{Re}\left(p^{n} z_{0}\right)\right), z_{0} \in \mathbb{C},\left|z_{0}\right|=$ $1, p=e^{-j 2 \pi f}, f \in \mathbb{R} \backslash \mathbb{Q}$, it is:

$g_{\infty}(\mathbf{b})=\lim _{n \rightarrow \infty} \sup _{|z|=1} \operatorname{Re}\left[\frac{z^{*} z_{0}}{n} \sum_{i=0}^{n-1} \operatorname{sgn}\left(\operatorname{Re}\left(p^{i} z_{0}\right)\right) p^{-i} z_{0}^{*}\right]=\frac{2}{\pi}$

In this case it is not necessary to impose the condition $\operatorname{sgn}\left(\operatorname{Re}\left(p^{i} z_{0}\right)\right) \neq 0$ because $g_{\infty}(\mathbf{b})$ is independent of $\arg \left(z_{0}\right)$ and, therefore, the maximum is global. Therefore, among those bounded sequences for which $g_{\infty}(\mathbf{b})$ exists, any $S^{3}$ sequence of frequency $f$ presents a global maximum. Of course, any other sequence taking different values in a finite number of elements will present the same maximum.

\section{EXAMPLES}

In this section we present two different case studies in which $S^{3}$ sequences are local optima. Case study 1 is related to the discrete-time actuation with global impulses of spiral waves in excitable media [15]-[16]. With this example it will be graphically shown how it is that $S^{3}$ sequences are locally optimal for the actuation of resonator-like systems. Case study 2 is a variant of the usual PDO topology.

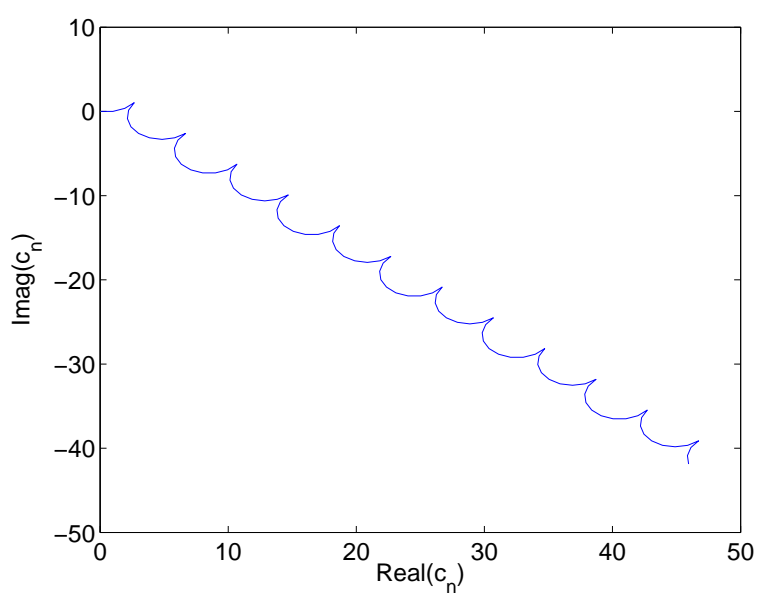

Fig. 2. Simulation of system (56) with $f=1 / 17, \alpha=1, z_{0}=1+j$ and initial condition $c_{0}=0$.

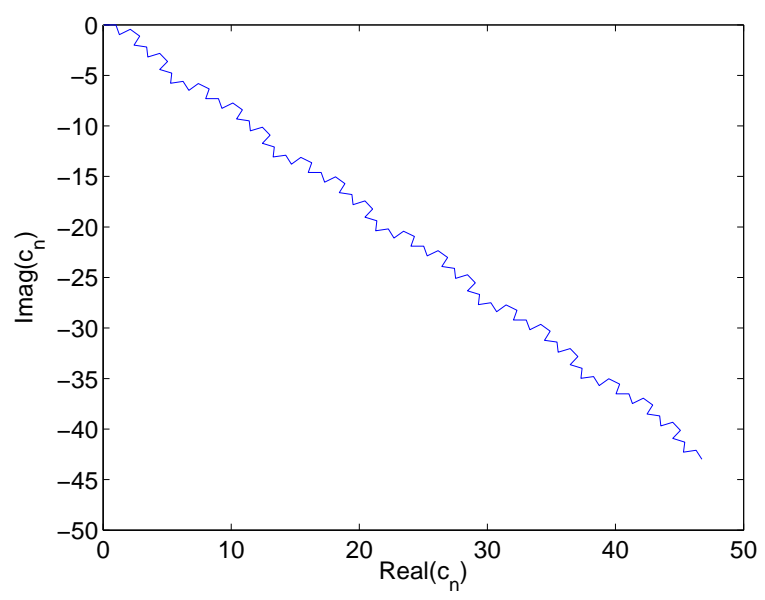

Fig. 3. Simulation of system (56) with $f=5 / 17, \alpha=1, z_{0}=1+j$ and initial condition $c_{0}=0$.

\section{A. Case study 1}

Let us consider a system defined as:

$$
c_{n+1}=c_{n}+b_{n} \alpha^{n} p^{n}
$$

for $n>0$ where $c_{0}$ is the initial condition and $p=e^{j 2 \pi f}, f \in \mathbb{Q}, \alpha \leq 1$. The application of the results of the previous section shows that sequences of the type $b_{n}=\operatorname{sgn}\left(\operatorname{Re}\left(p^{n} z_{0}\right)\right), z_{0} \in \mathbb{C}$ provide a locally unique maximum of $\left|c_{n}-c_{0}\right|$, for a sufficiently high $n$, among all bounded sequences $\left(b_{n}\right):\left|b_{n}\right| \leq 1$.

Figures 2 and 3 show the trajectories of this system actuated by $S^{3}$ sequences. It can be seen in Figure 2 that the trajectory is formed by semicircles glued in the form of 'm's, and that in Figure 3 the points in the circle that at each time mark the direction of the next step have experienced a permutation with respect to the case of Figure 2, reflecting in fact equation (69) of the Appendix. This is because in the first case the numerator of the frequency is 1 , whereas in 


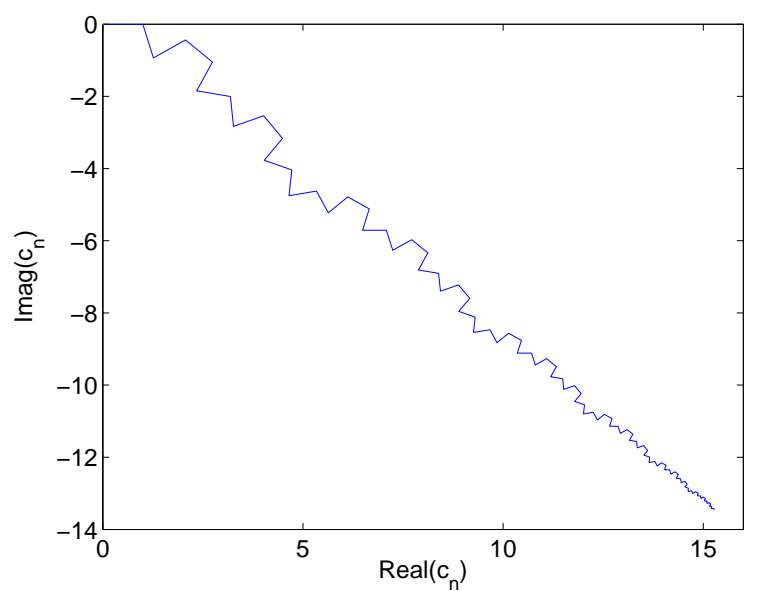

Fig. 4. Simulation of system (56) with $f=5 / 17, \alpha=0.97, z_{0}=1+j$ and initial condition $c_{0}=0$.

the other it is 5 . Finally, Figure 4 shows a case in which $\alpha<1$.

The displacement direction in the lossless systems $(\alpha=$ 1) can be calculated with expressions (28) or (29), taking into account that $\operatorname{sgn}\left(\operatorname{Re}\left(p^{n} z_{0}\right)\right)=\operatorname{sgn}\left(\operatorname{Re}\left(p^{-n} z_{0}^{*}\right)\right)$ and therefore:

$$
\begin{gathered}
\lim _{n \rightarrow \infty} \arg \left(c_{n}-c_{0}\right)=\lim _{n \rightarrow \infty} \arg \left(c_{n+N}-c_{n}\right)= \\
=\left\{\begin{array}{lc}
\frac{\pi}{2}-\frac{\pi}{N}-\frac{2 \pi\lfloor\beta N\rfloor}{N}, & N \text { even } \\
\frac{\pi}{2}-\frac{\pi}{2 N}-\frac{2 \pi\lfloor 2 \beta N\rfloor}{2 N}, & N \text { odd }
\end{array}\right.
\end{gathered}
$$

with $\beta=1 / 4-\frac{\arg \left(z_{0}^{*}\right)}{2 \pi}$. This angle is approximately $\arg \left(z_{0}^{*}\right)$, for large $N$.

1) Comparison of case study 1 with a $\Sigma-\Delta$-like actuation: In the previous example, the excitation being applied to $c_{n}$ follows the rotations of the circle $\left(p^{n}\right)$. Applying the results of section III, we apply a negative excitation each time that $\operatorname{sgn}\left(\operatorname{Re}\left(p^{n} z_{0}\right)\right)=-1$. This means that, if the phase of the vector $p^{n} z_{0}$ is $\Phi_{n}=n 2 \pi f+\arg \left(z_{0}\right)$, we apply a negative feedback force each time $\Phi_{n}$ lies in $(\pi / 2,3 \pi / 2)$. In the case we were only allowed to apply positive or null impulses, then each time the excitation would be applied in $(\pi / 2,3 \pi / 2)$ we would apply no excitation (waiting to excite it in the other half of the circle).

Another possible strategy would be to apply excitation at time $n$ if a given angle $(\theta)$ was between $\Phi_{n-1}$ and $\Phi_{n}$. Therefore:

$$
\begin{gathered}
b_{n}=f+\left\langle\frac{\Phi_{0}-\theta}{2 \pi}+(n-1) f\right\rangle-\left\langle\frac{\Phi_{0}-\theta}{2 \pi}+n f\right\rangle \\
c_{n+1}=c_{n}+b_{n} \alpha^{n} p^{n} z_{0}
\end{gathered}
$$

where $\Phi_{0}=\arg \left(z_{0}\right), p=e^{j 2 \pi f}$, and for a given $z_{0} \in \mathbb{C}$. In this case the bitstream has exactly the form of a $\Sigma-\Delta$

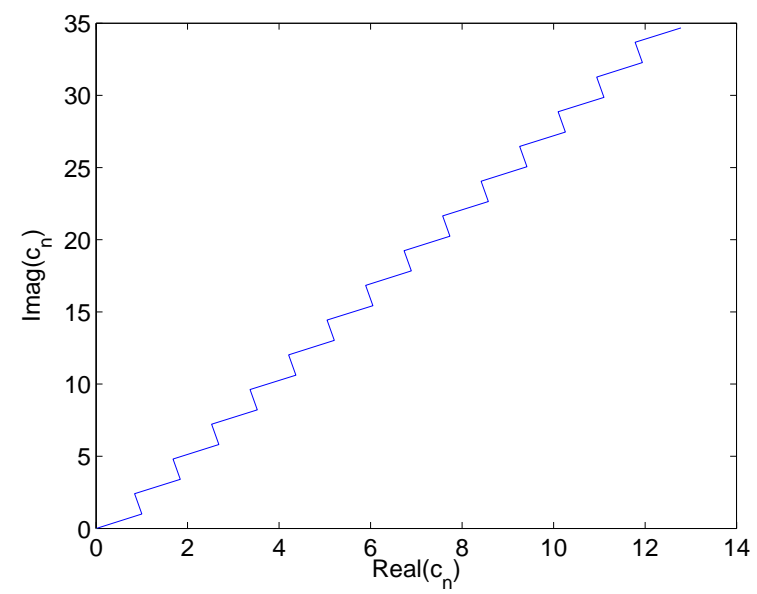

Fig. 5. Simulation of system (58) with $f=2 / 7, \alpha=1, z_{0}=1+j, \theta=0$ and initial condition $c_{0}=0$.

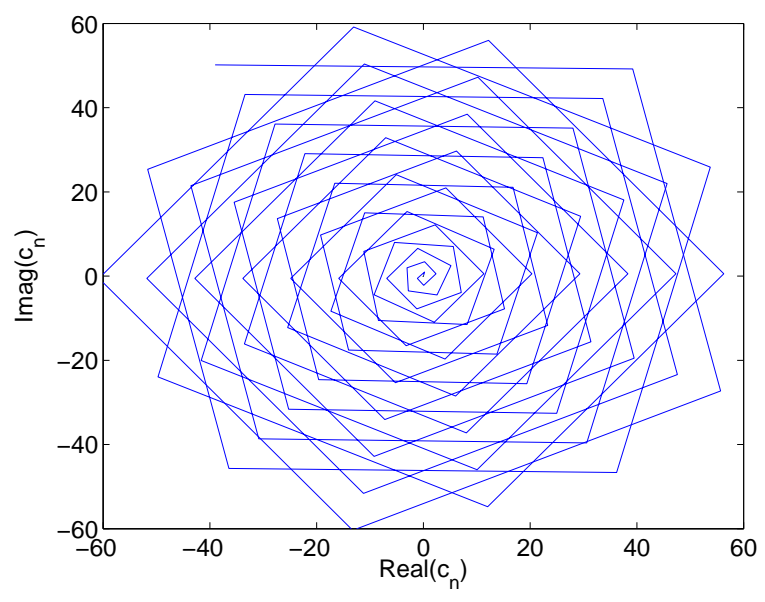

Fig. 6. Simulation of system (59) with $f=3 / 14, \alpha=1, z_{0}=1+j$ and initial condition $c_{0}=0$.

first-order modulator [17]-[18]. Figure 5 shows a simulation of a system of the type in (58).

\section{B. Case study 2}

Let us now consider a system defined as:

$$
c_{n+1}=\alpha p\left(c_{n}+b_{n}\right)
$$

In this case, it is:

$$
c_{n+k}-\alpha^{k} p^{k} c_{n}=(\alpha p)^{k} \sum_{i=0}^{k-1} \alpha^{-i} p^{-i} b_{n+i}
$$

and this term has the form of equation (25). In order to maximize $\left|c_{n+k}-p^{k} c_{n}\right|$ as shown in Section III, we will use an $S^{3}$ sequence, $b_{n}=\operatorname{sgn}\left(\operatorname{Re}\left(p^{n} z_{0}\right)\right), p=e^{j 2 \pi f}$. Figure 6 shows the result of this actuation for a system governed by equation (60) with $\alpha=1$. The result in this case is very similar to the trajectories of a lossless PDO [4] tuned to the frequency of the resonator. This will lead us in the next section to propose a variant of the usual topology of PDOs. This oscillator will 
always be tuned to rational frequencies in the lossless case [4].

\section{A DIFFERENT PDO TOPOLOGY}

\section{A. Direct PDOs}

In previous sections we have seen that $S^{3}$ sequences are optimal for certain types of systems, basically those in which the function to maximize has the form of equation (12). PDOs under certain conditions provide $S^{3}$ sequences [4]. The time evolution of a PDO, with $G(z)=z^{-L}$ as feedback filter, is described by:

$$
u_{n+1}=p u_{n}-j \delta \operatorname{sgn}\left(\operatorname{Re}\left(u_{n-L}\right)\right)
$$

where $\delta \in \mathbb{R}, p \in \mathbb{C}, u_{n} \in \mathbb{C}$ (note that now $|p|$ may be different of 1 ). The usual interpretation with a mechanical resonator is that the PDO senses information on the position of the resonator and applies a delta of force after $L$ clock cycles. This force is translated into an instantaneous change in the velocity of the resonator. Usually the real part of the complex variable $u_{n}$ is the position of the resonator and its imaginary part is the velocity. It can be easily checked that:

$$
u_{k+n}-p^{n} u_{k}=-j \delta p^{n-1} \sum_{i=k}^{k+n-1} p^{-i} \operatorname{sgn}\left(\operatorname{Re}\left(u_{i-L}\right)\right)
$$

Therefore PDOs are systems such that $u_{k+n}-p^{n} u_{k}$ has the form of system (25). On the other hand, it is shown in [4] that in order to obtain $S^{3}$ sequences at the output of the PDO, in the case of a lossless resonator with rational frequency, it is necessary that the oscillator is 'tuned' to this frequency. If a PDO is 'tuned' to a rational frequency, then conditions (31) and (32), are automatically satisfied for $G_{N}=u_{k+N}-u_{k}, k \geq 0$, at least for certain initial conditions of the resonator ([4]).

The reason to have frequencies to which the PDO is not 'tuned' is due to the fact that each time the oscillator applies a delta of force to the resonator, it is not guaranteed in general that $\operatorname{sgn}\left(\operatorname{Im}\left(u_{n}\right)\right)$ coincides with the sign of the applied excitation, which depends on the position, after a number of delays. In order to guarantee the coincidence we may define a variation of the typical PDO topology on which:

$$
u_{n+1}=p\left(u_{n}+\delta \operatorname{sgn}\left(\operatorname{Re}\left(u_{n}\right)\right)\right)
$$

or

$$
u_{n+1}=p\left(u_{n}+j \delta \operatorname{sgn}\left(\operatorname{Im}\left(u_{n}\right)\right)\right)
$$

For convenience, we use the first expression (63). This variant of the PDO will be called DPDO (Direct PDO). With this circuit, at each sampling time we must sense the sign of the variable that is instantly changed by the application of the delta of force. If we apply an excitation of the same sign $(\delta>0)$ we are increasing the energy of the resonator, and for the other sign $(\delta<0)$ we are stopping the resonator (unless the resonator is near the origin, $\left|u_{n}\right|<\delta$ ). This fact can be used in some applications [5]. In practical realizations a few nanoseconds delay can be present.

Theorem 3: If a lossless DPDO of frequency $f=\frac{M}{N}$, g.c.d. $(M, N)=1$ has an initial condition $u_{0}$ such that $\left|\operatorname{Re}\left(p^{n} u_{0}\right)\right|>n|\delta|, 0 \leq n<N$, then $\operatorname{sgn}\left(\operatorname{Re}\left(u_{n}\right)\right)=\operatorname{sgn}\left(\operatorname{Re}\left(p^{n} u_{0}\right)\right)$.

Proof. Following a similar procedure as in [4], we will calculate the sequence:

$$
h_{n}\left(u_{0}\right)=u_{n}-p^{n} u_{0}=\sum_{i=0}^{n-1} p^{n-i} \operatorname{sgn}\left(\operatorname{Re}\left(p^{i} u_{0}\right)\right)
$$

A sufficient condition for the sequence $b_{n}=$ $\operatorname{sgn}\left(\operatorname{Re}\left(p^{n} u_{0}\right)\right)$ to be a solution of the oscillator is that $\operatorname{sgn}\left(\operatorname{Re}\left(p^{n} u_{0}+h_{n}\left(u_{0}\right)\right)\right)=\operatorname{sgn}\left(\operatorname{Re}\left(p^{n} u_{0}\right)\right)$ [4].

Now, using the fact that $p^{N}=1$, for any given $n \geq 0$ :

$$
h_{n}\left(u_{0}\right)=h_{n \bmod N}\left(u_{0}\right)+\left\lfloor\frac{n}{N}\right\rfloor p^{n} h_{N}\left(u_{0}\right)
$$

and taking into account that $\left|h_{n \bmod N}\left(u_{0}\right)\right| \leq|n \delta|$, together with that fact that $\operatorname{sgn}\left(\operatorname{Re}\left(p^{n} h_{N}\left(u_{0}\right)\right)\right)=\operatorname{sgn}\left(\operatorname{Re}\left(p^{n} u_{0}\right)\right)$, as was proved in Section III for $G_{N}$, we have that $\operatorname{sgn}\left(\operatorname{Re}\left(p^{n} u_{0}+h_{n \bmod N}\left(u_{0}\right)\right)\right)=\operatorname{sgn}\left(\operatorname{Re}\left(p^{n} u_{0}\right)\right)$, and therefore $\operatorname{sgn}\left(\operatorname{Re}\left(u_{n}\right)\right)=\operatorname{sgn}\left(\operatorname{Re}\left(p^{n} u_{0}\right)\right), n \geq 0$.

In the case of a leaky resonator, in which $p=\alpha e^{j 2 \pi f}, 0<\alpha<1$, the following result can be obtained.

Theorem 4: For any given frequency $f=M / N$, g.c.d. $(M, N)=1$, there is $0<\alpha_{1}<1$ such that for any leaky DPDO, with $1>|p|>\alpha_{1}, p=\alpha e^{-j 2 \pi f}$, there is a constant $C(p) \in \mathbb{R}^{+}$such that if $\left|\operatorname{Re}\left(p^{n} u_{0}\right)\right|>C(p), 0 \leq n \leq N-1$, then $\operatorname{sgn}\left(\operatorname{Re}\left(u_{n}\right)\right)=\operatorname{sgn}\left(\operatorname{Re}\left(p^{n} u_{0}\right)\right), n \geq 0$.

Proof. Following the same reasoning as in Theorem 3, we only need to prove that the sequence:

$$
h_{n}\left(u_{0}\right)=\sum_{i=0}^{n-1} p^{n-i} \operatorname{sgn}\left(\operatorname{Re}\left(p^{i} u_{0}\right)\right)
$$

is such that $\operatorname{sgn}\left(\operatorname{Re}\left(p^{n} u_{0}+h_{n}\left(u_{0}\right)\right)\right)=$ $\operatorname{sgn}\left(\operatorname{Re}\left(p^{n} u_{0}\right)\right), n \geq 0$. On the other hand, following a similar procedure as in Section IV-F, for any given rational frequency $f$, there is a $0<\alpha_{1}<1$, such that for $\alpha_{1}<|p|<1$ there is a $n_{0}(p)$ such that for all $n>n_{0}(p)$ it is $\operatorname{sgn}\left(\operatorname{Re}\left(p^{n} u_{0}\right)\right)=\operatorname{sgn}\left(\operatorname{Re}\left(h_{n}\left(u_{0}\right)\right)\right)$. Finally if $\left|\operatorname{Re}\left(p^{n} u_{0}\right)\right|>\frac{1-|p|^{n_{0}(p)}}{1-|p|} \geq \operatorname{Max}_{i=0}^{n_{0}(p)}\left|h_{i}\left(u_{0}\right)\right|, 0 \leq n \leq$ $n_{0}(p)$, then $\left|\operatorname{Re}\left(p^{n} u_{0}\right)\right|>\left|h_{n}\right|, 0 \leq n \leq n_{0}(p)$ and therefore $\operatorname{sgn}\left(\operatorname{Re}\left(p^{n} u_{0}+h_{n}\left(u_{0}\right)\right)\right)=\operatorname{sgn}\left(\operatorname{Re}\left(p^{n} u_{0}\right)\right), n \geq 0$.

It is worth noting that in contrast to the normal PDO case with a lossless resonator, it is not possible to get double spiral 
trajectories with DPDOs, because the oscillator is always tuned to any rational frequency.

\section{B. Energy considerations for DPDOs}

The purpose of this subsection is to analyze under which conditions DPDOs provide bitstreams that are solutions of Problem P2.

Theorem 5: For any given time $n \geq 0$, except for a set of initial conditions of the resonator of zero Lebesgue measure, DPDOs generate bitstreams that are locally unique solutions of problem P2 regardless of the initial condition of the resonator.

Proof. Let us consider a DPDO applying the excitation $b_{i}=\operatorname{sgn}\left(x^{\prime}\left(i T_{S}\right)\right)$. At each sampling time, a delta of force is applied and its sign coincides with the sign of the instantaneous velocity of the resonator. If $\operatorname{sgn}\left(x^{\prime}\left(i T_{S}\right)\right) \neq 0,0 \leq i<n$, the sequence provides a locally unique maximum of the total energy transferred to the resonator up to time $n$.

Now, we may define $T(u): \mathbb{R}^{2} \rightarrow \mathbb{R}^{2}$ as the transformation on the variables of the resonator determined by the DPDO and $L_{0}=\left\{(x, v) \in \mathbb{R}^{2}: v=0\right\}$. The result is finally proved taking into account that $\bigcup_{i=0}^{\infty} T^{-i}\left(L_{0}\right)$ is a set of zero Lebesgue measure.

\section{CONCLUSIONS}

The problem of finding optimal pulsed actuations to obtain maximum energy transfer or amplitude in linear resonators has been analyzed. Three different problems have been established: maximization of the final energy of a resonator after $n$ deltas of force have been applied, maximization of the energy delivered to the resonator and maximization of the resulting amplitude. Under some conditions $S^{3}$ sequences at the resonant frequency of the resonator may be optimal for some of the problems. In particular, for lossless resonators, $S^{3}$ sequences provide asymptotically locally unique maxima of the three problems. Finally, a new PDO topology has been proposed that, except for a set of initial conditions of the resonator of zero Lebesgue measure, provides a locally unique maximum in energy transfer to resonators.

\section{ACKNOWLEDGEMENTS}

The author wishes to thank Prof. Frank Hoppensteadt, Courant Institute, NYU, for his useful comments, and the anonymous reviewer who kindly provided the proof of section II.B. This work was supported by a CICYT TEC200767951/MIC project.

\section{APPENDIX}

$G_{N}$ for an $S^{3}$ sequence of frequency $f=\frac{M}{N}, \alpha=1$

Given an $S^{3}$ sequence of the form $b_{n}=\operatorname{sgn}\left(\operatorname{Re}\left(p^{n} z_{0}\right)\right)$, with $p=e^{-j 2 \pi f}, z_{0} \in \mathbb{C}$ and g.c.d. $(M, N)=1$ it is:

$$
b_{n}=2(\langle n f+1 / 2+\beta\rangle-\langle n f+\beta\rangle), n \geq 0
$$

where $\beta=\frac{1}{4}-\frac{\arg \left(z_{0}\right)}{2 \pi}$, and $G_{N}$ has been defined as $G_{N}=\sum_{i=0}^{N-1} \alpha^{-i} p^{-i} b_{i}=\sum_{i=0}^{N-1} p^{-i} b_{i}$, because $\alpha=1$.

Now, due to the fact that g.c.d. $(M, N)=1$, it is for any $y \in \mathbb{R},[4]:$

$$
\begin{aligned}
& \left\{\left\langle i \frac{M}{N}+y\right\rangle: i=0, \cdots, N-1\right\}= \\
= & \left\{\frac{i}{N}+\frac{\langle y N\rangle}{N}: i=0, \cdots, N-1\right\}
\end{aligned}
$$

and:

$$
\begin{aligned}
& \sum_{i=0}^{N-1}\left\langle i \frac{M}{N}+y\right\rangle e^{j 2 \pi \frac{M}{N} i}=e^{-j 2 \pi y} \sum_{i=0}^{N-1} \frac{i}{N} e^{j 2 \pi \frac{i+\langle y N\rangle}{N}}= \\
= & e^{-j 2 \pi \frac{\lfloor y N\rfloor}{N}} \sum_{i=0}^{N-1} \frac{i}{N} e^{j 2 \pi \frac{i}{N}}=-\frac{j}{2} e^{-j \frac{\pi}{N}} \frac{e^{-j 2 \pi \frac{\lfloor y N\rfloor}{N}}}{\sin \frac{\pi}{N}}
\end{aligned}
$$

Therefore, for $N$ even, and taking into account that $\lfloor(\beta+$ $1 / 2) N\rfloor / N=\lfloor\beta N\rfloor / N+1 / 2$, it is:

$$
G_{N}=j 2 e^{-j 2 \pi \frac{\lfloor\beta N\rfloor}{N}} \frac{e^{-j \pi / N}}{\sin \frac{\pi}{N}}
$$

For $N$ odd, and taking into account that for $\langle\beta N\rangle \in[0,1 / 2)$ it is $\lfloor\beta N\rfloor / N=\lfloor\beta 2 N\rfloor / 2 N$, and that for $\langle\beta N\rangle \in[1 / 2,1)$ it is $\lfloor\beta N\rfloor / N=\lfloor\beta 2 N\rfloor / 2 N-1 / 2 N$, following the same procedure it is possible to reach expression (29).

\section{REFERENCES}

[1] Jiangfeng Wu; Carley, L.R.; Electromechanical S-D modulation with high-Q micromechanical accelerometers and pulse density modulated force feedback, IEEE Trans. on Circuits and Systems I, Vol.53, No. 2, p. 274-287, 2006.

[2] Jun Su Lee, Lucyszyn, S.; A Micromachined Refreshable Braille Cell, Jornal of Microelectromechanical Systems, Vol. 14, No. 4, p. 673-682, 2005.

[3] Dominguez, M.; Pons-Nin, J.; Ricart, J.; Bermejo, A.; Costa, E.F.; Morata, M.; Analysis of the $\Sigma-\Delta$ pulsed digital oscillator for MEMS, IEEE Trans. on Circuits and Systems I, Vol. 52, No. 11, p. 2286-2297, 2005.

[4] M. Dominguez, J. Pons, J. Ricart, General dynamics of Pulsed Digital Oscillators, IEEE Trans. on Circuits and Systems I, Vol. 55, No. 7, pp. 2038-2050, 2008.

[5] M. Dominguez, J. Pons, J. Ricart, Application of Pulsed Digital Oscillators in 'reverse mode' to eliminate undesired vibrations in high-Q MEMS resonators, Proc. ISCAS 2007, pp. 925 - 928, 2007.

[6] J. Ricart, J. Pons, M. Dominguez, A. Rodriguez, E. Figueras, M.C. Horrillo, J. Gutierrez, I. Sagayo, Application of Pulsed Digital Oscillators to Volatile Organic Compounds Sensing, Sensors and Actuators: B. Chemical, in print.

[7] Dominguez, M.; Pons-Nin, J.; Ricart, J.; Figueras, E.; The MEMS pulsed digital oscillator (PDO) below the Nyquist limit, Sensors and Actuators A: Physical, Sensors and Actuators A: Physical, Vol. 136, No. 2, pp. 690-696, 2007. 
[8] M. Dominguez, J. Pons, J. Ricart, J. Juillard, E. Colinet Linear analysis of the influence of FIR feedback filters on the response of the pulsed digital oscillator, Analog Integrated Circuits and Signal Processing, in print.

[9] US Patent 7009458 - Method and system for fast wake-up of oscillators, 2006.

[10] E. Sontag, Mathematical Control Theory, Springer-Verlag 1998.

[11] D. P. Bertsekas, Nonlinear Programming, Athena Scientific, 1999.

[12] G.M. Lee, N.N. Tam, N.D. Yen, Quadratic programming and affine variational inequalities, Springer-Verlag, 2005

[13] O.L. Mangarasian, Locally unique solutions of quadratic programs, linear and and nonlinear complementarity problems, Mathematical Programming, Vol. 19, p. 200-212, 1980.

[14] A. Bellow, V. Losert, The weighted pointwise ergodic theorem and the individual ergodic theorem along subsequences, Transactions of the American Mathematical Society, Vol. 288, No. 1, pp. 307-345, 1985.

[15] V. S. Zykov, H. Engel, Unified approach to feedback-mediated control of spiral waves in excitable media, Analysis and Control of Complex Nonlinear Processes in physics, Chemistry and Biology, pp. 243-272, World Scientific, Singapore, 2007.

[16] A. Karma, V.S. Zykov, Structure of the Resonance Attractor for Spiral Waves in Excitable Media, Phys. Rev. Lett., Vol. 83, 2453-2456, 1999.

[17] Gray, R.M. Oversampled Sigma-Delta Modulation, IEEE Transactions on Communications, Vol. 35, p. 481-489, 1987.

[18] Gray, R.M. Spectral analysis of quantization noise in a single-loop sigma-delta modulator with DC input, IEEE Transactions on Communications, Vol. 37, p. 588-599, 1989. 